\title{
Invención de problemas contextualizados de probabilidad: una competencia por desarrollar en profesores de Matemáticas
}

\author{
Máster Lorena Salazar Solórzano' ${ }^{1}$, Universidad Nacional de Costa Rica y \\ Universidad de Costa Rica \\ Recibido: $\quad 6$ de julio, 2017 \\ Aceptado: 31 de octubre, 2017
}

\section{Resumen}

Los actuales programas de estudio de matemáticas en Costa Rica incluyen, por primera vez, el tema de probabilidad en todos los niveles, razón por la cual los profesores deben ser competentes, no solo en esa área del saber, sino también en la creación de problemas que sean contextualizados y suficientemente motivadores para introducir este tema. Con el interés de responder a esta demanda nacional se da este estudio, para desarrollar la competencia de invención de problemas de probabilidad en futuros profesores de matemática. La muestra seleccionada fue de 10 profesores en formación de la Universidad Nacional de Costa Rica, a los que se les proporcionó material didáctico concreto, así como datos del Compendio de Estadísticas del Informe del Estado de la Nación (2016), para crear sus problemas. Para ello se siguieron cuatro etapas estratégicas: análisis de enunciados de problemas clásicos de probabilidad, variación y adaptación de problemas, invención de problemas propios, evaluación y mejora de estos. Los resultados muestran cómo, con la implementación de estas cuatro fases junto con el apoyo de material económico y datos nacionales de libre acceso, se logra, de una manera expedita, problemas potentes y contextualizados, que empoderan al futuro docente en la competencia de invención de problemas, lo que da lugar, tanto a problemas lúdicos interesantes (juegos), como a aquellos relacionados con problemáticas nacionales que conciencian a los jóvenes costarricenses.

\section{Abstract \\ Creating math problems on Probability: a competency to be developed among math teachers}

For the first time, the topic of probability will be included in every level of the current Costa Rican mathematics study program, which should be reason enough for teachers to be competent not only in probability itself, but also in the creation of problems related to that subject, as well as great motivators to introduce that topic to students. In an effort to respond to the national demand in this field, this course will be held with the main purpose of developing a competition to create math problems associated with probability among future math teachers. A sample of ten teachers still pursuing a major in Mathematics Teaching at the Costa Rican National University (UNA) was taken, who were then given suitable teaching material, as well as a Compilation of Statistics of the State of the Nation Report (Costa Rica, 2016) to create math problems. For this purpose, four strategic stages were set up: analysis in classical probability, variation, and statement adaptation, creation of new mathematical problems and evaluation/improvement of what was created. Results show that by implementing these four stages, offering financial support and free access to data at a national level, powerful mathematical contextual problems can quickly be accomplished that will empower the mathematics problem creation competency in future teachers, allowing not only for entertaining and interesting problems but also for raising awareness of other mathematics-related issues amongst young Costa Ricans.

1 Lorena Salazar es máster en Matemáticas, graduada de la Universidad de Toledo, Ohio, Estados Unidos. Labora en la Universidad de Costa Rica y en la Universidad Nacional, como docente de la Escuela de Matemáticas e investigadora. Contactos: lorena.salazarsolorzano@ ucr.ac.cr y lorena.salazar.solorzano@una.cr.
Lorena Salazar Solórzano. Invención de problemas contextualizados de probabilidad: una competencia a desarrollar en profesores de matemática. Revista Comunicación. Año 38, volumen 26, número 2, julio-diciembre, 2017. Instituto Tecnológico de Costa Rica. ISSN: 0379-3974 / e-ISSN1659-3820.

\section{PALABRAS CLAVE:}

Creación de problemas, resolución de problemas, probabilidad, material lúdico, profesores de matemáticas, educación matemática.

\section{KEY WORDS:}

math problems creation, solving math problems, probability, ludic material, math teachers and math education. 


\section{INTRODUCCIÓN}

Los actuales currículos de Matemáticas, generados por el Ministerio de Educación Pública de Costa Rica (MEP) en 2012 incluyen -por primera vez en todos los niveles- el tema de Probabilidad, una de las áreas con gran aplicabilidad en la vida cotidiana. Según Batanero (2006):

(...) el azar está presente en la vida cotidiana, existen muchos contextos en los que aparecen nociones de incertidumbre, riesgo y probabilidad, por ejemplo, el pronóstico del tiempo, diagnóstico médico, estudio de la posibilidad de tomar un seguro de vida o efectuar una inversión, evaluación de un estudiante, etc. (p. 3).

Esta reforma curricular exige que los profesores tengan, no solo un dominio sólido en probabilidad, sino que además desarrollen competencias para elegir y crear problemas Ilamativos a sus alumnos. Con el interés de satisfacer esta demanda nacional, se realiza este estudio, dentro del marco del proyecto de investigación Pry01-1011-2015, a cargo de quien escribe: "Desarrollo e implementación de metodologías alternativas a la tradicional en los cursos de matemática para futuros profesores de secundaria".

Muchos docentes en ejercicio indican tener debilidades en los temas de probabilidad laplaciana, probabilidad condicional, teorema de Bayes y ley de los grandes números (Meneses, Mora, Peña y Gómez, 2013). Sin embargo, aunque en su formación universitaria (en Costa Rica) recibieron un curso básico de probabilidad y estadística (al menos los docentes de secundaria, puesto que en general, los de primaria no reciben ningún curso al respecto), estos conceptos han sido probablemente olvidados, dado que por mucho tiempo no se han visto en la necesidad de formar a los estudiantes en este tema. Estas deficiencias se agravan cuando se trata de la didáctica de la probabilidad y cómo enseñarla eficientemente, ya que esta preparación estuvo ausente en su formación inicial.

En la actualidad, muchos profesores de secundaria y maestros de primaria, sedientos de material didáctico para explicar esta materia, muchas veces toman problemas de libros de texto de otros países, descontextualizados, con un lenguaje que dificulta la comprensión de los enunciados de los problemas y de poco interés para los estudiantes costarricenses. Es por eso que esta investigación intenta desarrollar en los futuros docentes, la competencia de crear sus propios problemas, necesaria en cualquier profesor. Para ello, se tomó como contexto temático la probabilidad, en el que manifiestan debilidad, según encuesta realizada por Meneses, Mora, Peña y Gómez (2013), con el fin de darles empoderamiento al respecto y seguridad en las aulas.

En esta investigación se plantearon las siguientes preguntas: ¿cómo lograr que los futuros profesores de matemática sean capaces de crear problemas de probabilidad potentes y llamativos? ¿Cómo empoderarlos en la invención de problemas para introducir el tema de probabilidad de una manera agradable al estudiante?

Después de definir las preguntas de investigación, se buscó crear problemas lúdicos con algunos juegos para introducir los conceptos de probabilidad, de modo que resultaran agradables a los estudiantes. Según Salvador (2007),

(...) una clase con un juego es una sesión motivada desde el comienzo hasta el final, produce entusiasmo, interés, desbloqueo y gusto por estudiar matemáticas. Un juego bien elegido puede servir para introducir un tema, ayudar a comprender mejor los conceptos o procesos, afianzar los ya adquiridos, adquirir destreza en algún algoritmo o descubrir la importancia de alguna propiedad, reforzar automatismos y consolidar un contenido.

En una segunda fase, se les proporcionó datos reales de estadísticas y censos nacionales, de modo que los futuros profesores crearan problemas de realidades costarricenses, que además se prestaran a reflexiones con los educandos. La Tabla 1 muestra lo que Batanero (2013), señala como tareas y problemas que pueden resolver los estudiantes según la edad, los cuales se tomaron en cuenta a la hora de crear los problemas. 
Invención de problemas contextualizados de probabilidad: una competencia por desarrollar en profesores de Matemáticas

Tabla 1: Tareas que se pueden realizar por edad

\begin{tabular}{lll}
\hline Estrategia & Edad & Descripción \\
\hline Estrategias aditivas & $\begin{array}{l}8-11 \\
\text { años }\end{array}$ & $\begin{array}{l}\text { Elegir la caja donde la diferencia entre casos favorables } \\
\text { y desfavorables sea mayor. Se toman en cuenta todos los } \\
\text { datos, pero no se usan proporciones. }\end{array}$ \\
\hline $\begin{array}{lll}\text { Estrategia de } \\
\text { correspondencia }\end{array}$ & $\begin{array}{l}12-13 \\
\text { años }\end{array}$ & $\begin{array}{l}\text { Establecer la proporción entre el número de casos } \\
\text { favorables y desfavorables en una de las cajas y comparar } \\
\text { con la composición de la otra, eligiendo la caja que dé } \\
\text { mayor proporción. Resuelve el problema correctamente } \\
\text { cuando el número tiene una proporción sencilla (por } \\
\text { ejemplo 2 a 1) de casos favorables y desfavorables en } \\
\text { una de las cajas, pero no en la otra. }\end{array}$ \\
\hline $\begin{array}{l}\text { Estrategias } \\
\text { multiplicativas }\end{array}$ & $\begin{array}{l}\text { Aplicar la regla de Laplace. Compara las fracciones } \\
\text { formadas por los números de casos favorables y } \\
\text { desfavorables en las urnas; resuelve todos los problemas } \\
\text { de este tipo. }\end{array}$ \\
\hline
\end{tabular}

Fuente: Batanero, C. (2013). La comprensión de la probabilidad en los niños: ¿qué podemos aprender de la investigación? Atas do III Encontro de probabilidades e estatistica na escola. Braga: Centro de Investigaçao em Educaçao da Universidade do Minho.

De acuerdo con esta información, se decidió dirigir el estudio de creación de problemas, a un nivel de secundaria, es decir, para estudiantes mayores de13 años. Por otro lado, se decidió darles estadísticas reales para crear problemas relacionados con problemáticas nacionales, los cuales los adolescentes estén en condición de comprender y concienciar.

\section{METODOLOGÍA}

Para esta investigación se usó como contexto temático el de combinatoria elemental y la regla de Laplace. El estudio se aplicó en una muestra de 10 estudiantes de la carrera Enseñanza de la Matemática de la Universidad Nacional de Costa Rica, quienes estaban llevando, con la misma docente e investigadora, un curso de resolución de problemas. Previo a la experiencia, se les asignaron dos lecturas que debían discutir y luego exponer a sus compañeros sobre creación de problemas (Malaspina, 2013; Michaela, Ellerton y Cai, 2015), con el objetivo de prepararlos para la actividad. Luego se les proporcionó material didáctico concreto, así como datos del Compendio de Estadísticas del Informe del Estado de la Nación (2016), para crear sus problemas. Para la recolección de información se usó un diario, donde se fue anotando todo lo que ocurrió en el aula y se recogieron evidencias escritas (las modificaciones y problemas creados por los grupos de trabajo).

\section{Etapas para la invención de problemas}

Se siguieron cuatro fases para que los profesores en formación lograran plantear sus problemas: análisis de enunciados de problemas clásicos de probabilidad, variación y adaptación de problemas, invención de problemas propios, evaluación y mejora de estos. Dichas etapas han sido usadas y validadas en otras diferentes experiencias en áreas de análisis y álgebra, como puede verse en Salazar (2015), con lo que se obtuvo resultados positivos. Se fundamentan en la variación de problemas para paulatinamente dar lugar a nuevas creaciones, incluyendo una etapa previa en la que se analizan problemas dados por expertos (autores de libros o problemas de la web) y otra etapa posterior, donde después de la invención de problemas, se persigue su mejora, después de una evaluación utilizando criterios de calidad previamente definidos. Las etapas, de acuerdo con Salazar (2016), se describen en la Tabla 2: 
Tabla 2: Etapas para creación de problemas

\begin{tabular}{|c|c|}
\hline Etapas & Descripción \\
\hline $\begin{array}{l}\text { I Etapa: Análisis de } \\
\text { enunciados: }\end{array}$ & $\begin{array}{l}\text { Leer y reflexionar sobre los enunciados de problemas y ejercicios } \\
\text { propuestos en libros de texto (intención del autor, el orden seguido, } \\
\text { nivel de dificultad, redacción). }\end{array}$ \\
\hline $\begin{array}{l}\text { Il Etapa: Variación de } \\
\text { problemas: }\end{array}$ & $\begin{array}{l}\text { Realizar variaciones a los problemas de la etapa anterior, cambiando } \\
\text { algunas hipótesis, modificando algunos de los requerimientos, } \\
\text { contextualizándolos a entornos familiares a los estudiantes meta. }\end{array}$ \\
\hline $\begin{array}{l}\text { III Etapa: Creación de } \\
\text { problemas propios: }\end{array}$ & $\begin{array}{l}\text { Crear sus propios problemas, ya sea producto de variaciones de } \\
\text { variaciones del problema original, o problemas completamente } \\
\text { independientes. }\end{array}$ \\
\hline $\begin{array}{l}\text { IV Etapa: Evaluación y de } \\
\text { problemas creados: }\end{array}$ & $\begin{array}{l}\text { Realizar una evaluación de los problemas por pares, creados con } \\
\text { miras a su mejoramiento, en redacción, presentación. }\end{array}$ \\
\hline
\end{tabular}

Salazar, L. (2016). Creación de problemas mediante un proceso guiado de cuatro etapas. Revista del Congreso Internacional de Docencia Universitaria e Innovación (CIDUI), 3.

\section{DESCRIPCIÓN DE LA EXPERIENCIA}

A continuación, se hace una descripción de la experiencia y cómo los profesores de matemática en formación, fueron generando nuevos problemas vía variaciones.

\section{Creación de problemas usando material lúdico}

Es bien sabido que las instituciones de secundaria costarricenses, rara vez dan apoyo económico para que el docente pueda adquirir material didáctico para impartir sus clases. Es por eso que en la actividad se eligió un material económico y de fácil acceso (se venden en tiendas populares). Con esto se busca que el futuro docente pueda inventar problemas motivantes y lúdicos sin que esto le signifique un gran costo en dinero. Para ello se puso a disposición de los futuros profesores de matemática, una mesa con diferentes materiales lúdicos (dados, ruleta, bolas, bolsas, alfombritas y diferentes juegos de mesa), de modo que pudieran elegir alguno para inventar problemas de probabilidad elemental, como se muestra en la figura 1:

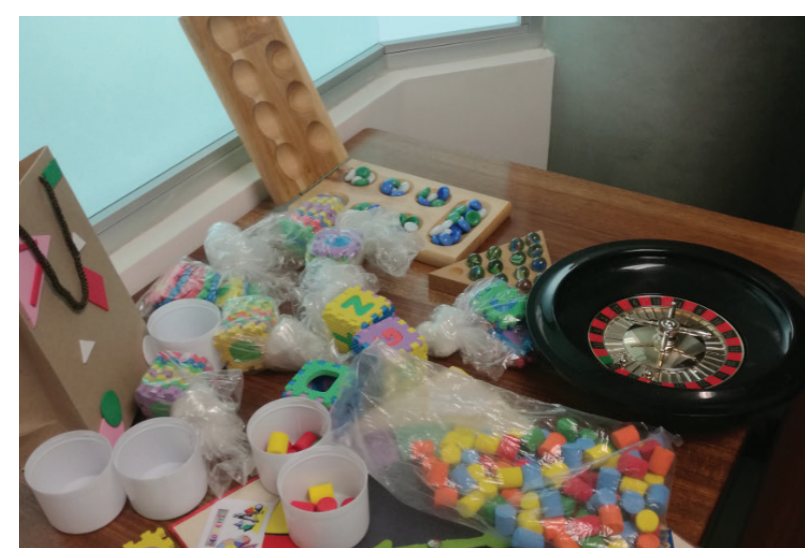

Figura 1. Material usado en la actividad. Fuente propia.

A continuación, se ilustran algunos ejemplos de los problemas creados por los diferentes grupos de trabajo, usando un material muy versátil y económico. 
Tabla 3: Problemas creados por los participantes

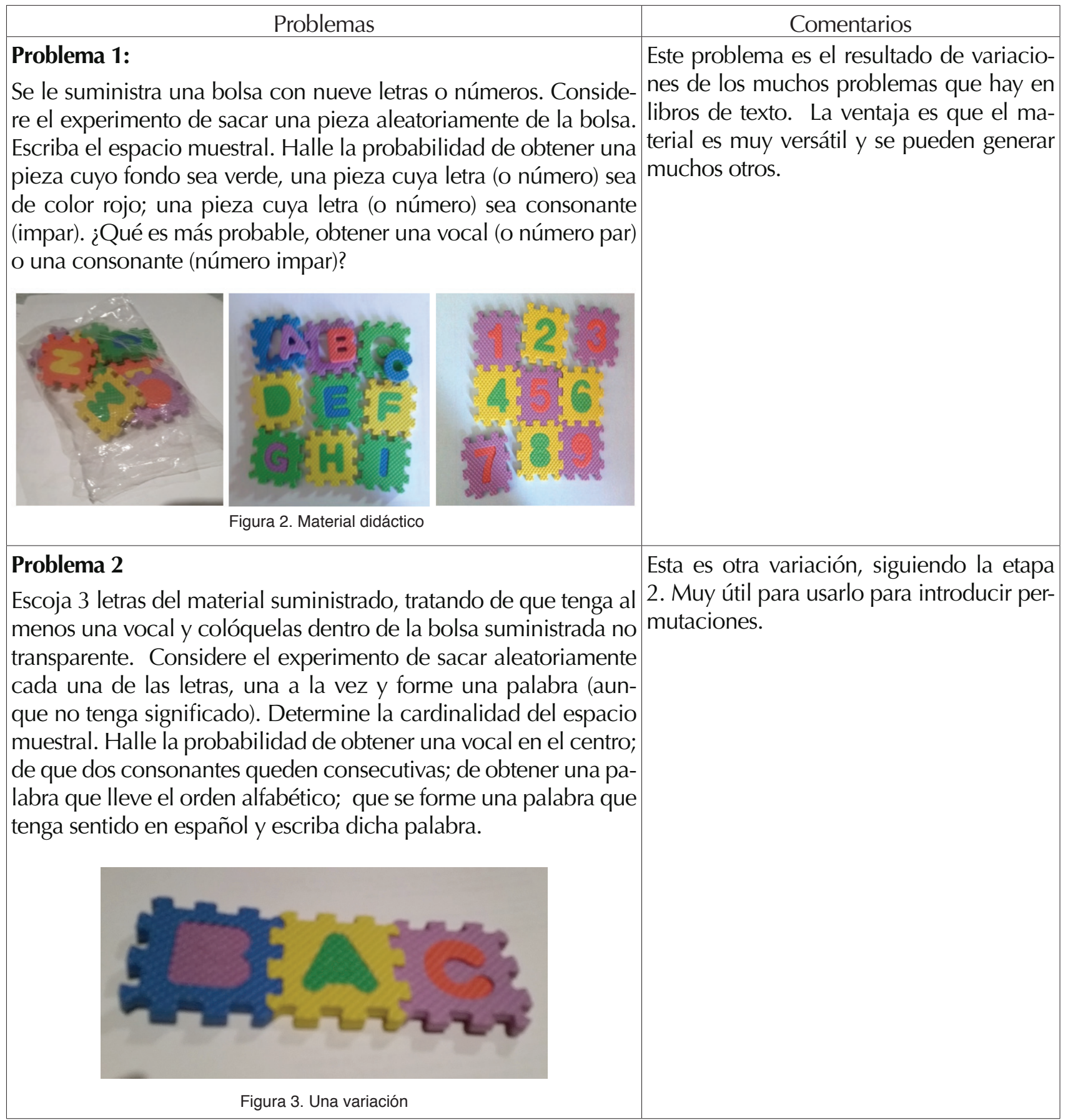

Se presentan tres juegos, planteados por los estudiantes, los cuales surgen vía variaciones de los problemas previos, es decir, realizando variaciones de las variaciones anteriores. Es interesante cómo la invención de problemas, da pie a que la mente genere sobre este, otro problema, con dificultad y potencialidades mayores. 
Tabla 4: Problemas creados por los participantes

\begin{tabular}{|l|}
\hline \multicolumn{1}{|c|}{ Problemas tipo juegos } \\
\hline Problema 3: \\
Escoja ahora 6 de las piezas de su alfom- \\
brita y forme un dado con ellas, pegando \\
las piezas. Considere el experimento de \\
lanzar el dado una vez. Determine el es- \\
pacio muestral. Halle la probabilidad de \\
que salga una vocal; de que salga una \\
consonante. iCuál sería el espacio mues- \\
tral si se lanzan dos dados a la vez? Use el \\
dado suyo y del compañero.
\end{tabular}

tes probabilidades: obtener la letra que corresponde a la cara superior del dado hueco; que la letra sacada rellene alguna de las caras laterales del dado hueco; que la letra sacada rellene alguna de las caras de color lila.

\section{Problema 5:}

El siguiente juego es para dos personas que deben seguir las siguientes reglas: El jugador 1 lanza un dado tradicional. Si el resultado es un número par, tiene el derecho de rellenar una de las caras del dado hueco con la letra correspondiente y volver a lanzar el dado tradicional. En caso contrario, le toca el turno a su contrincante. Gana el primero que logre rellenar completamente el dado.

Determine la probabilidad de rellenar todas las caras del dado de letras en seis lanzadas seguidas del dado tradicional, es decir la probabilidad de que alguien gane el juego sin dejar jugar al otro. Determine la probabilidad de ganar el juego sin dejar jugar al otro, si en lugar de un dado se lanzan dos dados y se considera que la suma sea par.

Figura 5. Variación del juego anterior

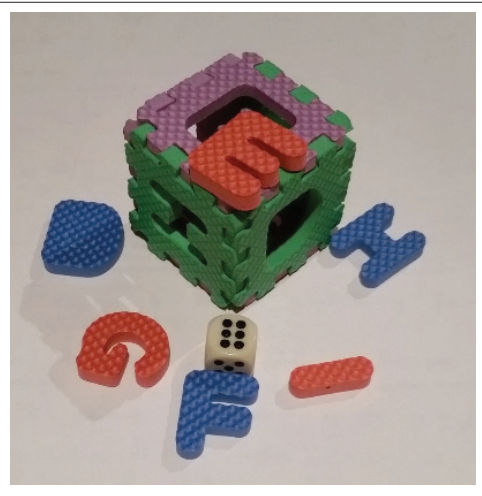

Figura 6. Variación de la variación del juego anterior
Comentarios

Este es un primer juego con el que podrían introducirse conceptos preliminares de probabilidad. Es una variación de un dado normal de números de 1 a 6, con la diferencia de que cada estudiante podría tener un dado diferente, de acuerdo con las fichas que le correspondió.

Esta es una variación de la anterior, con la diferencia de que incluye un grado más de dificultad. En el fondo es el mismo principio de lanzar dos dados y que coincidan en el resultado obtenido. Pero con este material, el estudiante puede motivarse más. Es interesante cómo surgen las ideas realizando variaciones de variaciones anteriores.

Este es otro juego que involucra dos personas, incorporando otro factor: la competitividad como un elemento motivante extra. Observe que involucra el dado de letras y otro dado normal. El grado de dificultad es mayor, ya que la pregunta involucra composición de probabilidades (en el sentido de realizar varias tareas), una seguida de otra. Es interesante resolverlo con el principio de multiplicación y por la simple regla de Laplace, poner a los estudiantes a comparar las soluciones y que, por supuesto, ellos aprendan, jugando. 


\section{Creación de problemas usando estadísticas na- cionales reales}

Finalmente, se les asignó como tarea buscar datos oficiales de Estadísticas y Censos de Costa Rica, para crear problemas de probabilidad que se presten a reflexiones de los estudiantes, acerca de datos reales y sobre todo, de las razones posibles de lo que indican esos datos y la probabilidad.

\section{Figura 7. Costa Rica: Población de 15 años o más por nivel de instrucción según zona, región de planificación y sexo, julio 2016}

\begin{tabular}{|c|c|c|c|c|c|c|c|c|c|c|c|}
\hline \multicolumn{12}{|c|}{$\begin{array}{l}\text { CUADRO } 3 \\
\text { Costa Rica: Población de } 15 \text { añ }\end{array}$} \\
\hline \multirow{3}{*}{$\begin{array}{l}\text { Zona, región de } \\
\text { planificación y sexo }\end{array}$} & \multirow{3}{*}{ Total } & \multicolumn{10}{|c|}{ Nivel de instrucción } \\
\hline & & \multirow{2}{*}{$\begin{array}{c}\text { Sin } \\
\text { instrucción }\end{array}$} & \multicolumn{2}{|c|}{ Primaria } & \multicolumn{2}{|c|}{ Secundaria académica } & \multicolumn{2}{|c|}{ Secundaria técnica } & \multicolumn{2}{|c|}{ Educación superior } & \multirow[b]{2}{*}{ Ignorado } \\
\hline & & & Incompleta & Completa & Incompleta & Completa & Incompleta & Completa & \begin{tabular}{c|} 
Pregrado y \\
grado
\end{tabular} & Posgrado & \\
\hline & & & & & & & & & & & \\
\hline Central & 2463754 & 60046 & 230787 & 576832 & 520542 & 371411 & 41887 & 52932 & 541046 & 66799 & 1472 \\
\hline Hombres & 1166985 & 29004 & 95808 & 284904 & 253891 & 174623 & 21327 & 26741 & 246967 & 33068 & 652 \\
\hline Mujeres & 1296769 & 31042 & 134979 & 291928 & 266651 & 196788 & 20560 & 26191 & 294079 & 33731 & 820 \\
\hline Chorotega & 285989 & 11572 & 37756 & 67294 & 62771 & 46100 & 6314 & 4697 & 45948 & 3319 & 218 \\
\hline Hombres & 140258 & 6300 & 17537 & 35843 & 31790 & 22214 & 2957 & 2530 & 19266 & 1603 & 218 \\
\hline Mujeres & 145731 & 5272 & 20219 & 31451 & 30981 & 23886 & 3357 & 2167 & 26682 & 1716 & \\
\hline Pacífico Central & 217176 & 12206 & 32591 & 55206 & 48548 & 25248 & 8708 & 4654 & 27924 & 2002 & 89 \\
\hline Hombres & 105527 & 7219 & 16288 & 28828 & 23377 & 11236 & 3437 & 2547 & 11837 & 758 & \\
\hline Mujeres & 111649 & 4987 & 16303 & 26378 & 25171 & 14012 & 5271 & 2107 & 16087 & 1244 & 89 \\
\hline Brunca & 268577 & 14996 & 43211 & 73972 & 53980 & 30794 & 6800 & 6347 & 35397 & 2964 & 116 \\
\hline Hombres & 129071 & 7739 & 20155 & 37066 & 27218 & 13451 & 3744 & 2971 & 15888 & 839 & \\
\hline Mujeres & 139506 & 7257 & 23056 & 36906 & 26762 & 17343 & 3056 & 3376 & 19509 & 2125 & 116 \\
\hline Huetar Caribe & 316994 & 16787 & 57878 & 89524 & 71411 & 37029 & 7007 & 5486 & 29430 & 2085 & 357 \\
\hline Hombres & 153751 & 9273 & 29139 & 45567 & 34832 & 17537 & 2546 & 2200 & 11372 & 928 & 357 \\
\hline Mujeres & 163243 & 7514 & 28739 & 43957 & 36579 & 19492 & 4461 & 3286 & 18058 & 1157 & \\
\hline
\end{tabular}

Fuente: Informe Estado de la Nación. (2016). Compendio de Estadísticas del Informe del Estado de la Nación. Recuperado de http://www.estadonacion.or.cr/estadisticas-index.

Este tipo de datos, pueden utilizarse para involucrar al estudiante en situaciones reales de su entorno social y darle herramientas para una reflexión guiada que pueda complementarse en otras asignaturas, como educación cívica, estudios sociales, entre otras. Por ejemplo, en el caso particular presentado en el Cuadro 3, puede darse una rica reflexión sobre las diferencias en educación entre las zonas centrales y las rurales o una discusión sobre las posibles causas y soluciones. Esto da pie a formar ciudadanos críticos y conscientes de las diferencias sociales. Este tipo de estadísticas son de libre acceso y hay gran cantidad de datos en diferentes temas que el docente puede utilizar como insumo para crear problemas interesantes a sus estudiantes, como los siguientes: 
Tabla 5: Problemas creados por los participantes

\begin{tabular}{|c|c|}
\hline Problemas & Comentarios \\
\hline $\begin{array}{l}\text { Problema 6: } \\
\text { ¿Cuál es la probabilidad de que, si se escoge una mujer al azar de } \\
\text { la zona central, esta tenga secundaria académica completa? ¿Y que } \\
\text { tenga educación superior? ¿Qué es más probable, escoger un hom- } \\
\text { bre o una mujer con educación universitaria completa del Pacífico } \\
\text { Central? ¿A qué cree que se debe esta situación? ¿Qué es más prob- } \\
\text { able, una mujer con secundaria completa de la zona central o de la } \\
\text { zona Huetar Caribe? ¿A qué cree usted que se debe esto? }\end{array}$ & $\begin{array}{l}\text { Es importante cómo los estudiantes in- } \\
\text { volucran preguntas en las que se presta } \\
\text { a la reflexión sobre el porqué de estas } \\
\text { diferencias a encontrar en los resultados } \\
\text { de las probabilidades solicitadas. }\end{array}$ \\
\hline $\begin{array}{l}\text { Problema 7: } \\
\text { Se desea escoger al azar dos personas para un trabajo específico. } \\
\text { ¿Cuál es la probabilidad de que ambas personas sean mujeres de } \\
\text { la región Chorotega con educación superior completa? ¿Cuál es la } \\
\text { probabilidad de que, una de las personas sea mujer y otro hombre, } \\
\text { y que ambos sean de la zona del Caribe? ¿Cuál es la probabilidad } \\
\text { de que, al menos una de las personas escogidas sea de la zona } \\
\text { Brunca? ¿Y de la zona central? ¿A qué cree que se debe esto? }\end{array}$ & $\begin{array}{l}\text { Otro conjunto de problemas creados, } \\
\text { en donde se involucra el factor laboral } \\
\text { con respecto a la educación. Terminan } \\
\text { con una consulta de a qué se debe este } \\
\text { hecho. }\end{array}$ \\
\hline
\end{tabular}

\section{CONCLUSIONES}

Los resultados de este estudio fueron positivos en cuanto a los productos o creaciones de problemas que los futuros docentes inventaron y sus alcances. Al inicio, los profesores en formación presentaron dudas e inseguridades sobre el propósito de la tarea, dado que nunca antes se habían enfrentado a ejercicios de este tipo, sino más bien a actividades de resolución de problemas. Sin embargo, a medida que palparon el material concreto y trabajaron con las estadísticas, las ideas fueron surgiendo paulatinamente, creando cada vez problemas con mayores potencialidades.

La incorporación de las cuatro etapas usadas en la invención de problemas, les llevó de una manera expedita a crear nuevos problemas. La primera etapa, que es la de explorar problemas de libros de texto, realizados por expertos, se desarrolló con muy buenos resultados, ya que tener el material en la mano, les dio a los futuros docentes insumos para realizar modificaciones y adaptaciones para generar nuevos problemas e incluso juegos, con los que ellos mismos indicaban la finalidad que se les podría dar. La segunda etapa se dio de manera natural, pues al inventar un problema, casi inmediatamente les daba ideas para generar alguna modificación, y crear otro problema, usualmente con mayor dificultad. Para la tercera etapa, la de creación de problemas originales, podría decirse que se llegó a un nivel relativamente bajo. Sin embargo, para una primera experiencia, no se podría decir que estuvo mal. De hecho, los juegos 3 , 4 y 5 son muy interesantes y motivadores. Esta es una competencia que se logra poco a poco y se refuerza con la práctica.

En la última etapa, que era la discusión de los problemas con miras a las mejoras, hubo interesantes reflexiones sobre lo que debía ser un buen problema y la importancia de la redacción y claridad de los requerimientos. Al respecto se hicieron cuestionamientos a problemas de libros de texto, en los que se dificultaba mucho saber qué se pedía, precisamente por la confusión que genera la redacción de algunos de ellos.

En el desarrollo de la actividad, los participantes expresaron su aprobación en cuanto a cómo, con un material barato y versátil, se pudo lograr tantos resultados. Después de indagar en las estadísticas que están disponibles en la web, ellos mismos fueron dando ideas de maneras en que podrían usarlas para generar problemas, no solo de probabilidad, sino también de estadística. Finalmente, en la evaluación de la actividad, reconocieron que se sentían más empoderados que al principio. 
Se recomienda inducir a los profesores en formación y a los que están en ejercicio, a crear sus propios problemas, porque esto les genera empoderamiento y seguridad en las aulas. Un profesor motivado, logra motivar a sus estudiantes, un profesor convencido de un problema interesante, puede más fácilmente interesar a sus alumnos. Bien lo dice el proverbio chino: "Regala un pescado a un hombre y le darás alimento para un día, enséñale a pescar y lo alimentarás para el resto de su vida".

\section{REFERENCIAS BIBLIOGRÁFICAS}

Batanero, C. (2006). Razonamiento probabilístico en la vida cotidiana: Un desafío educativo. En P. Flores y J. Lupiáñez (Eds.), Investigación en el aula de matemáticas. Estadística y Azar. Granada: Sociedad de Educación Matemática Thales.

Batanero, C. (2013). La comprensión de la probabilidad en los niños: ¿qué podemos aprender de la investigación? Atas do III Encontro de probabilidades e estatistica na escola. Braga: Centro de Investigaçao em Educaçao da Universidade do Minho.

Informe Estado de la Nación. (2016). Compendio de Estadísticas del Informe del Estado de la Nación. Recuperado de http://www.estadonacion.or.cr/ estadisticas-index.
Malaspina, U. (2013). Nuevos horizontes matemáticos mediante variaciones de un problema. Unión, 35, 135-143.

Meneses, S., Mora, A., Peña, H. y Gómez, A. (2013). Unidad didáctica de probabilidad para la enseñanza secundaria (Memoria de Seminario de Graduación, Universidad de Costa Rica).

Michaela, F., Ellerton, N. y Cai, J. (2015). Mathematical problem posing from research to effective practice. Springer.

Ministerio de Educación Pública (MEP). (2012). Programas de Estudio en Matemáticas. República de Costa Rica. I y II Ciclo de la Educación Primaria, III Ciclo de Educación General Básica y Educación Diversificada.

Salazar, L. (2015). Problem posing as a didactic resource in formal mathematics courses to train future secondary school mathematics teachers. Journal of Technology and Science Education (JOTSE). Special Issue, 5(2), 64-74.

Salazar, L. (2016). Creación de problemas mediante un proceso guiado de cuatro etapas. Revista del Congreso Internacional de Docencia Universitaria e Innovación (CIDUI), 3.

Salvador, A. (2007). El juego como recurso didáctico en el aula de matemáticas. Universidad Politécnica de Madrid. Recuperado de http://www2. caminos.upm.es/Departamentos/matematicas/ grupomaic/conferencias/12.Juego.pdf

ANEXO

\section{Tabla 6: Problemas adicionales}

\section{Problema 8:}

Las reglas del juego consisten en que solo se puede mover un espacio hacia adelante o diagonalmente, conforme a su tipo de alfombra (número o letra), se tira el dado y tiene derecho a avanzar si en la cara superior del dado corresponde a un número par. El primero que termine en recorrer el tablero es el ganador. Los jugadores se rifan con cuál de las mini-alfombras van, si las de números o letras. Una vez distribuido, eligen de qué lado del tablero deben partir, como se muestra en la imagen. ¿Qué probabilidad tiene el jugador que eligió números de terminar antes? ¿Qué probabilidad tiene el jugador que eligió letras de terminar antes?

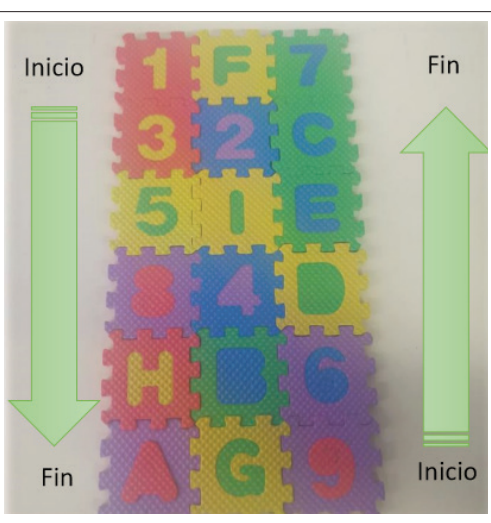

Figura 8. Otro juego 


\section{Problema 9:}

Se ha construido un cubo con MatsPuzzle en el que 1 de sus caras son vocales, 4 de sus caras son consonantes y la última cara son números. ¿Cuál es la probabilidad de que al lanzar este dado obtenga una cara con vocales? ¿Cuál es la probabilidad de que al lanzar este dado al aire se obtenga un número? ¿Cuál es la probabilidad de que al lanzar este dado al aire se obtenga la letra $k$ ?

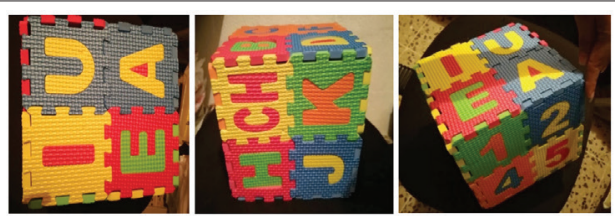

\section{Problema 10:}

Se disponen dos cajas con cilindros de colores. Si se quiere sacar un cilindro rojo, ¿cuál de las cajas debe escoger para tener mayor probabilidad de lograrlo?

\section{Problema 11:}

El juego de serpientes y escaleras es un tablero que cuenta con una cuadrícula de 100 cuadrados. En algunos de ellos se encuentran escaleras (bueno para el jugador) y en otras serpientes (malo para el jugador). Se inicia desde 0 y para avanzar se lanzan dos dados. Suponga que juegan dos estudiantes. El primer jugador lanza los dados. ¿Cuál es la probabilidad de que logre caer en la primera escalera? ¿En la segunda escalera? Suponga que un jugador logró subir por la segunda escalera hasta la posición 30. ¿Cuál es la probabilidad de que logre la escalera más grande? Si uno de los jugadores logró subir por la escalera más grande hasta la posición 84, ¿¿cuál es la probabilidad de que la serpiente más larga lo baje a la posición 24? Si un jugador está en la posición 7, ¿cuál es la probabilidad de que al lanzar los dados caiga en la siguiente serpiente? Si un jugador está en la posición 74, ¿cuál es la probabilidad de que logre ganar el juego en la siguiente lanzada de los dados?

\section{Problema 12:}

Se dispone de dos urnas, A y B, que contienen bolas de colores. La urna $\mathrm{A}$ tiene 7 bolas blancas, 2 bolas rojas y la urna B tiene 4 bolas blancas, 2 rojas y 2 verdes. Se lanza un dado. Si salen números impares, se extrae una bola de la urna A y si salen números pares, se extraen de la urna B. ¿Existe igual probabilidad de sacar una bola roja de cualquiera de las urnas? ¿Cuál es la probabilidad de que la bola extraída sea roja de la urna $\mathrm{A}$ ? Calcule la probabilidad de que la bola extraída sea verde. Si la bola extraída es blanca, ¿cuál es la probabilidad de que en el dado haya salido un número par? Ahora considere el experimento de extraer una bola de la urna A y reemplazarla por 2 bolas del otro color. Si se extrae una segunda bola, ¿cuál es la probabilidad de que la bola extraída sea roja? ¿Cuál es la probabilidad de que las 2 bolas extraídas sean del mismo color? ¿Si la segunda bola ha sido blanca, cuál es la probabilidad de que la primera también haya sido blanca?

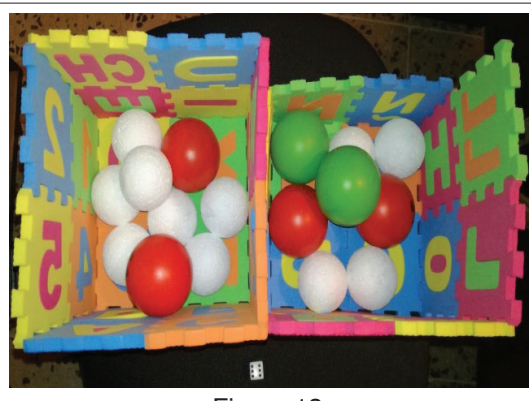

Figura 12.
Figura 11. Juego serpientes
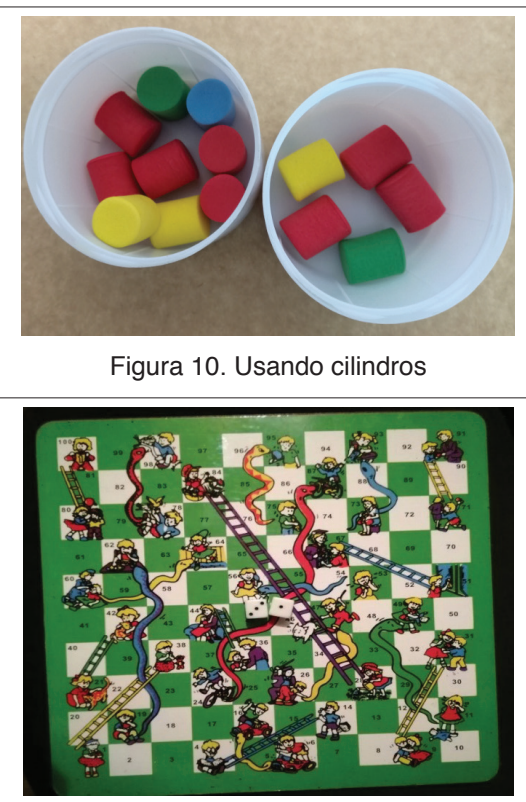

Figura 10. Usando cilindros

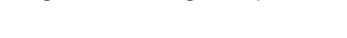




\section{Problema 13:}

Este es un juego de memoria que consta de 40 cartas, distribuidas en grupos numerados del 1 al 10 . Cada grupo posee 4 cartas con una imagen de un pez. El juego consiste en poner todas las cartas boca abajo e ir volteando una a la vez para encontrar parejas. ¿Cuál es la probabilidad de obtener un número del 1 al 10? ¿Cuál es la probabilidad de que al voltear una carta esta tenga un número par? ¿Cuál es la probabilidad de que al voltear una carta la próxima, sea una pareja?

\section{Problema 14:}

¿Cuál es la probabilidad de que, al girar la aguja central, esta apunte al color rojo, amarillo, rosado, azul, morado o celeste?

Problema 15:
¿Cuál es la probabilidad de que, al girar la ruleta, una de las
bolitas quede en el color rojo? ¿Cuál es la probabilidad de que
salga un número par o impar? ¿Cuál es la probabilidad de que
salga la potencia de un número al cuadrado?

